\title{
RARE PELVIC MASS - BLADDER ABSCESS
}

\author{
Vinoo Balakrishnan ${ }^{1}$
}

${ }^{1}$ Senior Consultant, Department of Gynaecology, Kerala Institute of Medical Sciences, Kerala.

\section{ABSTRACT}

\section{BACKGROUND}

Bladder wall abscesses as such are a rare clinical entity. In this case report, we are discussing the diagnostic challenges and operative difficulties of a pelvic mass which was presented clinically as an ovarian cyst.

\section{KEYWORDS}

Pelvic Mass, Bladder, Abscess, Neurogenic Bladder.

HOW TO CITE THIS ARTICLE: Balakrishnan V.Rare pelvic mass - bladder abscess. J. Evolution Med. Dent. Sci. 2016;5(104):76947695, DOI: $10.14260 / J e m d s / 2016 / 1735$

\section{BACKGROUND}

Bladder wall abscess has been reported only very rarely.(1) We present a case of bladder wall abscess which mimicked an ovarian cyst in a young girl who had meningomyelocoele and a neurogenic bladder. ${ }^{(2)}$

\section{CASE REPORT}

An 18-year-old girl was referred to emergency room from local hospital since they could not re-insert an indwelling Foley catheter.

She was a known case with meningomyelocoele operated soon after birth. She developed incontinence of urine and faeces and was found to have a low capacity spastic bladder. She was advised clean intermittent catheterisation (CIC) but shifted to indwelling catheter due to poor compliance. She suffered from recurrent UTI, obstructive nephropathy, secondary to poor compliance with CIC necessitating continuous indwelling catheter and chronic renal failure for which she was under the care of renal physician for last nine years.

A tender cystic mass arising out of pelvis reaching $2 \mathrm{~cm}$ above umbilicus was palpable and clinically thought to be distended bladder. During the attempt of catheterisation, even the inflation of the Foley's bulb was quite painful for the patient. Less than $20 \mathrm{~mL}$ of cloudy urine drained and pelvic mass persisted. Investigation showed azotaemia with creatinine of $110 \mathrm{mmol} / \mathrm{L}$ and low $\mathrm{Hb} 7.2 \mathrm{~g} \%$. Electrolytes were normal. Ultrasonography was done on next day. A midline thick walled cystic mass about $12 \mathrm{X} 11 \mathrm{~cm}$ in size was seen in the pelvis. Echogenic sediments were noted filling the cyst. Bladder was small and thick walled with catheter in situ. (See Fig 1). Uterus was compressed posteriorly and to the left. Ovaries could not be visualised as bladder was empty and could not be distended since it was painful. There was no evidence of fluid in the peritoneal cavity or cul-de-sac. Kidneys were enlarged and hydronephrotic.

Financial or Other, Competing Interest: None.

Submission 15-11-2016, Peer Review 16-12-2016,

Acceptance 22-12-2016, Published 29-12-2016.

Corresponding Author:

Dr. Vinoo Balakrishnan,

91/677, Tulips, Anyara PO,

Trivandrum-695029,

Kerala State, India.

E-mail: vinu9977@hotmail.com

DOI: $10.14260 /$ jemds/2016/1735
Limited helical computed tomography of the pelvis was done to evaluate the mass and the bladder. $5 \mathrm{~mL}$ of the diluted contrast was introduced through to the catheter and a few sections taken immediately. CT findings were consistent with sonographic findings and the large cystic mass in the pelvis was considered likely to be an ovarian cyst.

Laparotomy was done under antibiotic coverage. On opening the peritoneum, about 1.5 litres of thick pus extravasated from the lower part of the incision. A thickwalled abscess about $12 \times 14 \mathrm{~cm}$ seen encircling the bladder. Both dilated ureters were identified. Uterus, tubes and adnexae were separated from the cyst. Cyst wall separated by blunt and sharp dissection and the small contracted bladder was seen inside. Since the cyst wall was merged with the contracted bladder wall, a rim of the cyst wall was left near the bladder after attaining haemostasis. Post-operatively, she was treated with Amikacin and later changed to Gatifloxacin following culture reports. Histopathology showed chronic inflammatory lesion with abscess formation and layers of detrusor muscle surrounding abscess wall. Followup visit after eight weeks showed a clean wound but vesical capacity remained compromised.

\section{DISCUSSION}

Primary intravesical abscesses are rare.(3) Since this type of abscess has been reported in another patient with neurogenic bladder, there seems to be an association between the two entities. Here the systemic features of toxicity such as fever, malaise, nausea and vomiting were not profound since it was a longstanding issue. Local effect such as lower abdominal pain was profound. Since the bladder capacity was drastically reduced urinary frequency was quite prominent. The basic management of any abscess is the drainage and coverage with appropriate antibiotics. The choice of drainage include Ultrasound-guided aspiration and drainage or a CT-guided aspiration and drainage. But in our index case, the abscess was so large it needed to have laparotomy. In our patient we are unable to identify any aetiological factor, it may have been secondary to a bladder diverticulum or trauma on catheterisation. 


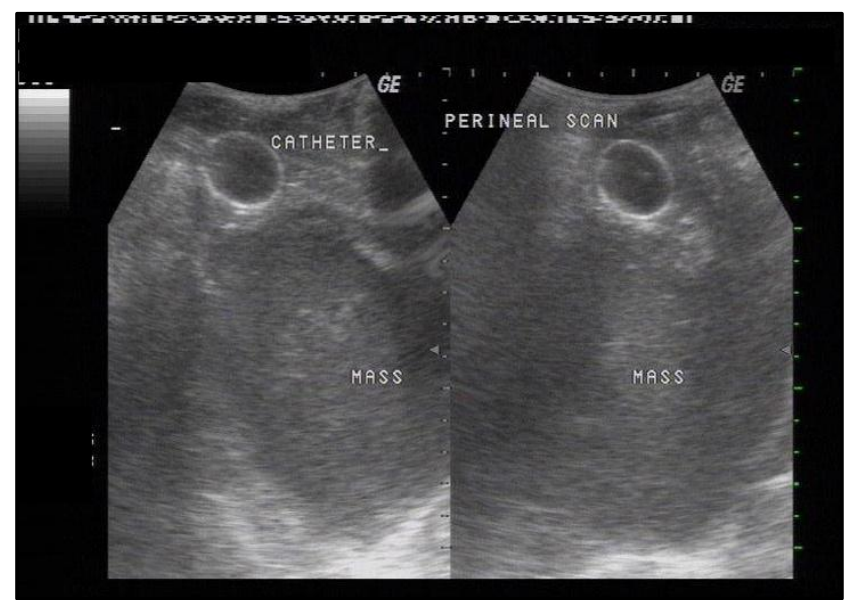

\section{REFERENCES}

1. Imamoto T, Tobe T, Mizoguchi K, et al. Perivesical abscess caused by migration of a fish bone from the intestinal tract. International journal of urology 2002;9(7):405-6.

2. Bamehriz F, Habib Z, Ahmed S. Bladder wall abscess in a girl with neurogenic bladder. Paediatric surgery international 2000;16(1):138-9.

3. Sarkar KK, Philp T, Thurly P. Intramural vesical abscess. British journal of Urology 1990;66(6):665.

Figure 1. Ultrasound Image 